\title{
DAS ÖSTERREICHER HEFT 2013
}

\section{Sehr geehrte Frau Kollegin, sehr geehrter Herr Kollege,}

wie jedes Jahr um diese Zeit darf ich Ihnen ein „Österreicher-Heft" vorstellen, da die Nummer 4 dieser Zeitschrift immer von KollegInnen der Österreichischen Gesellschaft für kontrollierte Akupunktur und TCM (OGKA) gestaltet wird. Frau Kollegin Birgit Richter schreibt diesmal über ein hochinteressantes und effektives Mikrosystem, die Hand- und Fußgelenksakupunktur, die sie unbedingt einmal probieren sollten. Die Kollegen Kaindlstorfer und Gasser schreiben mit mir über ein völlig neues System, die „Hypnoakupunktur“, welches die Vorteile der Kombination aus Akupunktur und Tiefenentspannung hervor streicht. Dr. Manfred Reininger hat wesentliche neue Erkenntnisse zum Störherdproblem aufzuzeigen und gibt Tipps, wie vor allem hartnäckige Störherde noch besser als bisher ausgeschaltet werden können. Kollegin StalzerBrunner gibt praxisnahe Tipps für die Therapie von Stress und Burn-Out, einer Problematik, die an Aktualität kaum zu überbieten ist. Das Autorenteam Pojer/Laber erweitert unseren Horizont in Sachen Master Tung Akupunktur bei Infertilität und Dysmenorrhoe. Kollege Gründler stellt das computergesteuerte Frequenzhämmerchen vor.

Ich darf Sie des Weiteren noch auf eine große Neuigkeit innerhalb der OGKA hinweisen: Eine neue Sektion der OGKA - die TCM Akademie für medizinische Fachkräfte - nimmt mit Jahreswechsel ihre Arbeit auf. Damit eröffnet sich für Sie die Chance, auch Ihre MitarbeiterInnen professionell in TCM ausbilden zu lassen und auf diese Weise noch kompetentere Helfer in Ihrer Ordination zu beschäftigen.Vielleicht wäre auch für Sie der eine oder andere Kurs von Interesse, denn im Rahmen der TCMAkademie unterrichten wir zum Beispiel Aromatherapie oder die 5-Elemente Ernährung.

Bitte informieren Sie sich unter www.tcmakademie.at.

Abschließend noch eine Idee: Es ist immer schwierig, wirklich sinnvolle Weihnachtsgeschenke für Mitarbeiter $\mathrm{zu}$ finden: Was halten Sie davon, heuer Gutscheine für einzelne Kurse oder eine ganze Ausbildung bei derTCMAkademie zu verschenken? Wir sind sicher, dass Ihre Mitarbeiter ein passendes Thema finden würden und dieses Geschenk würde der Qualität Ihrer Ordination zu Gute kommen. Wir beraten Sie gerne unter 0316-374050 um die beste Lösung für Sie und Ihre Mitarbeiter zu finden.

Abschließend bleibt mir nur noch Ihnen ganz herzlich ein frohes Weihnachtsfest, erholsame Festtage und ein gutes Neues Jahr 2014 zu wünschen! Somit verbleibe ich mit besten kollegialen Grüßen

Ihr

Prof. Dr. med. Leopold Dorfer

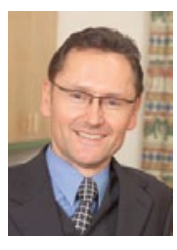

Prof. Dr. med. Leopold Dorfer

Präsident der Österreichischen Gesellschaft für Kontrollierte Akupunktur und TCM (OGKA)

Glacisstraße 7, A-8010 Graz

Tel. $+43316 / 374050$

E-Mail:office@ogka.at, Internet:www.ogka.at 